\title{
Probing the effect of tip pressure on fungal growth: Application to Aspergillus nidulans
}

\author{
Blanca González-Bermúdez, ${ }^{1,2}$ Qingxuan Li, ${ }^{1,2}$ Gustavo V. Guinea,${ }^{1,2,3}$ Miguel A. Peñalva ${ }^{4}$ and Gustavo R. Plaza ${ }^{1,2,5,}{ }^{*}$ \\ ${ }^{1}$ Center for Biomedical Technology, Universidad Politécnica de Madrid, E-28223 Pozuelo de Alarcón, Spain \\ ${ }^{2}$ Departamento de Ciencia de Materiales, ETSI de Caminos, Canales y Puertos, Universidad Politécnica de Madrid, E-28040 Madrid, Spain \\ ${ }^{3}$ Biomedical Research Networking Center in Bioengineering, Biomaterials and Nanomedicine (CIBER-BBN), Madrid, Spain \\ ${ }^{4}$ Departamento de Biología Celular y Molecular, Centro de Investigaciones Biológicas CSIC, Ramiro de Maeztu 9, E-28040 Madrid, Spain \\ ${ }^{5}$ Institute for Biomedical Engineering \& Nano Science, School of Medicine, Tongji University, Shanghai 200092, People's Republic of China
}

(Received 19 March 2017; published 8 August 2017)

\begin{abstract}
The study of fungal cells is of great interest due to their importance as pathogens and as fermenting fungi and for their appropriateness as model organisms. The differential pressure between the hyphal cytoplasm and the bordering medium is essential for the growth process, because the pressure is correlated with the growth rate. Notably, during the invasion of tissues, the external pressure at the tip of the hypha may be different from the pressure in the surrounding medium. We report the use of a method, based on the micropipette-aspiration technique, to study the influence of this external pressure at the hyphal tip. Moreover, this technique makes it possible to study hyphal growth mechanics in the case of very thin hyphae, not accessible to turgor pressure probes. We found a correlation between the local pressure at the tip and the growth rate for the species Arpergillus nidulans. Importantly, the proposed method allows one to measure the pressure at the tip required to arrest the hyphal growth. Determining that pressure could be useful to develop new medical treatments for fungal infections. Finally, we provide a mechanical model for these experiments, taking into account the cytoplasm flow and the wall deformation.
\end{abstract}

DOI: 10.1103/PhysRevE.96.022402

\section{INTRODUCTION}

In many important cellular phenomena, such as filamentous fungal invasion of substrates, pollen tube expansion in the transmitting tissue, and neuronal growth cone extension in animals, cellular growth proceeds by tip expansion. This type of growth, also known as apical or polarized growth, results in a rod-shaped elongation: a cylindrical protuberance finishing at the growing tip [1]. The importance of filamentous fungi as fermentation organisms is well exemplified by the estimate that $50 \%$ of the world's pharmaceutical and biotechnological products rely on these cells. Given also that some fungal cells are a common pathogen in many living organisms, there is a significant interest in understanding the mechanisms of tip growth [2,3]. In particular, the impact on human health of Aspergillus is well proven by the 20 human pathogens among them, such as A. fumigatus, which is life threatening in immune-compromised patients. The genus includes also beneficial species, specifically A. niger and A. oryzae, used to produce industrial enzymes and foodstuffs. Within this genus, A. nidulans is the most genetically amenable species and has proven useful for research of eukaryotic cell processes, for instance development, cytoskeletal function, DNA repair, and $\mathrm{pH}$ control [4]. Additionally, remarkable similarities to certain neuromuscular junctions are found in A. nidulans [5].

The polarized growth of fungal cells requires the synthesis of wall components in cellular organelles, fundamentally chitin and $\beta$-glucans, that are synthesized and sculpted by cell wall modifying enzymes. These proteins are transported by secretory vesicles to the cell tip and released through the plasma membrane via exocytosis [6]. Except for branching, which elicits new filamentous structures or hyphae, the site of

*Corresponding author: gustavo.plaza@upm.es growth is localized at a single region in each hypha [7]. While microtubules and actin microfilaments have been attributed an important role in regulating fungal tip growth $[8,9]$, the molecular machinery that regulates the exact sites of exocytosis remains unclear. Besides, the molecular mechanisms, such as stretch-activated calcium channels, by which the cell wall loosens to allow plastic deformation are still poorly identified [10,11].

Central to the function and development of living cells is mechanical deformability. Previous works have shown how mechanical deformability influences many biological processes in animal biology, such as migration of blood and immune cells [12], stem cell differentiation [13], and cancer [14], while in plant cells several studies have described the role of the mechanical properties of the wall in stability and resistance against pathogens [15]. In fungal cells and other tip-growing cells, some studies have reported different cell wall properties at the elongating tip from those in the lateral wall, showing how mechanical deformability accounts for the rod-shaped hyphae [16,17]. Accordingly, cell mechanics is crucial for understanding polarized growth.

Biophysical models of the polarized growth of cells with walls (plant, algal, and fungal cells) have been developed in the past decades to gain insight, organize, and evaluate the many fundamental aspects of the process (reviewed by Ortega and Welch [18]). The two coupled physical processes are (i) the water absorption from its surroundings via osmosis and (ii) the cell wall deformation as a consequence of the internal hydrostatic pressure that stresses the wall, the turgor pressure [7]. While the extension occurs, the incorporation of new materials to the wall is required to maintain a nearly constant thickness, preventing thinning and rupture of the wall. A comprehensive model for the hyphal growth would require taking into account metabolic aspects, intracellular transport, and mechanics of plastic stretching of the wall. While such 
a comprehensive model has not been developed, existing models are suited for the study of one of those processes: most of the models of a single hypha's growth concentrate on the biomechanical aspects involved in wall deformation $[19,20]$. Other works study the process of wall-components supply and are based on the concept of a vesicle supply center (VSC), where the carrier vesicles from the Spizenkörper, i.e., the organizing center of hyphal growth consisting of small vesicles located at the tip of the hypha, are transported with the materials required for the growth of the tip wall $[21,22]$. Additionally, recent works account for the anisotropic behavior of the wall components during the growth process [23].

Despite the available models, determining the mechanical parameters employed in the equations of the models represents a challenge for practical experimentation because of the small size of the cells and the nonhomogeneity of the cell wall. Among the plethora of techniques for the mechanical characterization of cells, only very few methods have been applied in fungal cells so far [24]. Atomic force microscopy (AFM), a technique based on the deflection of the tip of a flexible cantilever that indents a region of a cell, was employed to estimate the elastic modulus of the cell wall of fungal hyphae [2]. Another technique that has been used to measure the elastic properties of the wall of plant and fungal cell is the turgor-pressure probe [7]. This method consists of a glass microcapillary filled with an oil solution that is inserted into the cell. By measuring the amount of pressure required to remove the solution, the turgor pressure and the elastic properties can be assessed. A more recent approach to measure the wall properties of plant cells is based on custom-made microfluidic chips. Using these devices, some studies have related the geometry and the stiffness of the microchannels with the elasticity and pushing force of an elongating plant cell. However, local differences in the mechanical properties of the cell wall cannot be assessed with this technique. Moreover, in techniques such as optical or magnetic tweezers, the force range is too low to measure the mechanical parameters of cells with walls [15].

The turgor-pressure probe is useful to study the effect of the difference in pressure between the interior of the cell and the surrounding medium. In these experiments, the whole cell volume is affected and the whole cell wall is tested. It has not been developed to be used in very thin hyphae, as is the case in the species Aspergillus nidulans studied in this work, with a diameter of the order of $3 \mu \mathrm{m}$. To overcome these limitations, we have developed an experimental approach, tip-pressure experiments, based on the micropipette-aspiration technique. The procedure allows one to control locally the external pressure at the tip of the hypha. For cells with no wall, micropipette aspiration involves the aspiration of a cell by a microcapillary and the measurement of the aspirated length relative to a given suction pressure [25]. Due to the simplicity of the experimental device and the way the tests are carried out, micropipette aspiration is not as experimentally complex as other techniques, although the analysis of the measurements requires digital image processing and may be relatively time demanding and complicated to automatize [26]. We have adapted the technique by allowing fungal cells to grow into the microcapillary and controlling then the pressure inside the microcapillary.
Here, we investigate the polarized cell growth in the model species Aspergillus nidulans. The proposed tip-pressure technique may be used with other organisms. We have also developed a simple analytical model that incorporates the external pressure at the tip during the experiments. Our results show a correlation between the external pressure at the tip of the hypha and the growth rate, as predicted by the model. We consider that, using this procedure, systematic studies of the relation between growth rate and external pressure could provide new insights into the mechanics of hyphal growth and the effect of external pressure.

\section{EXPERIMENT}

\section{A. Preparation of polyacrylamide gel substrates}

In our experiments, we used a polyacrylamide (PAA) gel as substrate to grow the cells on its surface. This procedure allowed the successful manipulation of the microcapillaries without breaking them.

PAA gel is a common substrate used in cell-mechanics studies because the stiffness of the gel can be adjusted over the physiological range by varying the content of acrylamide and distilled water [27]. Prior to micropipette-aspiration tests, Aspergillus nidulans spores were cultured on a flexible PAA gel covered with collagen. The steps of the preparation procedure were the following:

(i) Activation of the glass to covalently bond the PAA gel: A $100 \mu$ l volume of $\mathrm{NaOH}(98 \%$, Panreac) was added onto a coverslip, and then wiped after $30 \mathrm{~min}$. Subsequently, $15 \mu \mathrm{l}$ of (3-aminopropyl)triethoxysilane (99\%, Sigma-Aldrich) were added and removed after $5 \mathrm{~min}$, then a $0.5 \%$ solution of glutaraldehyde (25\% in $\mathrm{H}_{2} \mathrm{O}$, Sigma-Aldrich) in phosphatebuffered saline (PBS) buffer was used to incubate the coverslips for $30 \mathrm{~min}$.

(ii) Synthesis of the PAA gels: $0.35 \mathrm{ml}$ of acrylamidebisacrylamide (19:1 ratio, $40 \% \mathrm{wt} / \mathrm{vol}$ solution, SigmaAldrich), $7 \mu \mathrm{l}$ of tetramethylethylenediamine (99\%, ReagentPlus), $23.2 \mu$ l of $10 \%$ ammonium persulfate solution $(99 \%$, Acros Organics), and $4.65 \mathrm{ml}$ of distilled water were used to prepare gels with $2.8 \%$ PAA. A volume of $250 \mu$ l of the mixture was deposited on the activated glass and covered with a small round cover glass ( $\varnothing 30 \mathrm{~mm}$ ) to obtain a flat gel surface.

(iii) Coating the gel with collagen: After $2-4 \mathrm{~h}$ to allow the gel polymerization, the cover glass was removed and a collagen solution was prepared using $4 \mu \mathrm{l}$ of type I collagen (4.89 mg/ml, Discovery Labware), $20 \mu \mathrm{l}$ of PBS, and 176 $\mathrm{ml}$ of distilled water. Subsequently, $50 \mu \mathrm{l}$ of the solution was added onto the surface of the gel and it was placed under UV light to sterilize for $45 \mathrm{~min}$.

(iv) Deposition of the spores solution: This process is described in the next section.

\section{B. Cell culture and experimental protocol}

Standard procedures were used throughout. An A. nidulans strain carrying a biA1 mutation that results in biotin auxotrophy was used as "wild type." This strain was maintained on complete medium [28], which was also used for the production of conidiospores. Conidiospores were resuspended in a solution of $0.0001 \%$ (wt/vol) Tween 20 and stored at $4{ }^{\circ} \mathrm{C}$. Experiments were carried out in watch minimal medium 


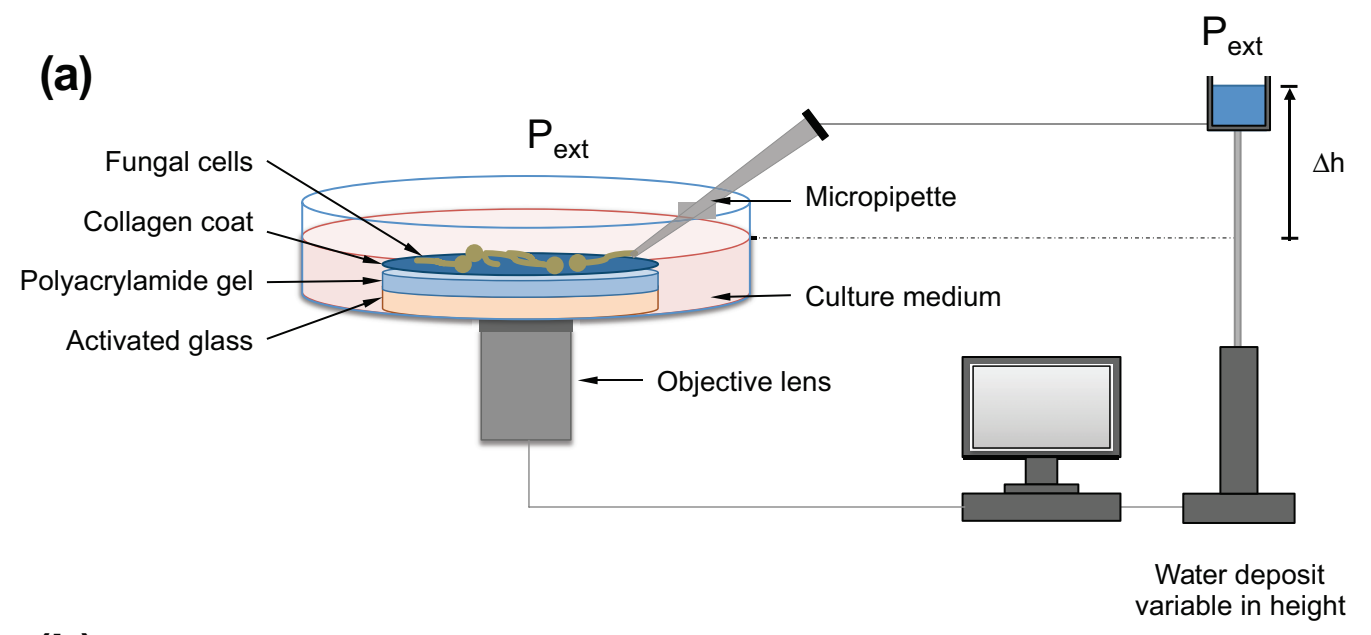

(b)

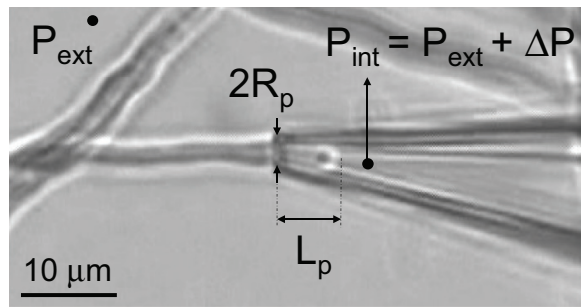

FIG. 1. (a) Sketch of the experimental procedure developed to perform tip-pressure experiments on A. nidulans. The differential pressure $\Delta P$ during the tip-pressure tests is proportional to the differential height of the water reservoir connected to the micropipette, $\Delta P=\rho g \Delta h$, where $\rho g$ is the specific weight of water. (b) Representative image of a tip-pressure experiment carried out on A. nidulans. The hyphal tip was tracked to quantify the growth rate in each pressure level.

(WMM) [29]. $1 \mu \mathrm{l}$ of this spore suspension was transferred to a sterile Eppendorf tube filled with $300 \mu$ l of WMM. As represented in Fig. 1(a), this diluted spore suspension was seeded on top of the collagen layer. Subsequently, the sample was incubated for $16-18 \mathrm{~h}$ at $26 \pm 1{ }^{\circ} \mathrm{C}$ prior to the tip-pressure experiments with the microcapillaries.

\section{Tip-pressure experiments}

Tip-pressure experiments were carried out using a microcapillary with a nominal internal diameter between 2 and $4 \mu \mathrm{m}$, which was connected to a distilled water reservoir [see Fig. 1(a)]. The experiments were conducted in a custom-built device [30], where the differential pressure $\Delta P$ was applied by a difference in height of the reservoir with respect to the cells, $\Delta h$. In this way, the pressure in the microcapillary was $P_{\text {ext }}+$ $\Delta P$, where $P_{\text {ext }}$ is the pressure in the growth medium containing the growing hypha. The cover-glass plate with the gel and the cells was placed in an optical Meiji TC5400 inverted microscope. During the cell growth process, the water flow in the microcapillary is negligible to produce a pressure drop due to frictional forces. Therefore, the differential pressure is given by $\Delta P=\rho g \Delta h$, where $\rho g$ is the specific weight of water.

In the experiments we tested, consecutively, various levels of differential pressure $\Delta P$, ranging from $\sim-800 \mathrm{~Pa}$ to $\sim 650 \mathrm{~Pa}$ (Fig. 2). Time-lapse images of the process were recorded as illustrated in Figs. 1(b) and 2(b).

\section{Measurements of tip growth}

Tip growth was defined as the change in hyphal tip position. Recorded images were analyzed to measure the length in the microcapillary, $L_{p}$. We used IMAGEJ to analyze the images acquired in each experiment, computing the growth rate, $d L_{\mathrm{p}} / d t$, by fitting a straight line to the experimental values in the plot of $L_{\mathrm{p}}$ versus $t$.

\section{RESULTS AND DISCUSSION}

\section{A. Experimental results}

Aiming to develop an experimental procedure to study the mechanics of hyphal growth and of tissue invasion, we worked on a system to perform tip-pressure experiments by controlling the external pressure applied locally at the tip of hyphae, allowing the simultaneous measurement of the internal length of the cell.

We used a microcapillary connected to a water reservoir [26], as schematized in Fig. 1, to apply a differential pressure $\Delta P$ between the interior of the microcapillary and the growth medium. Inside and outside the microcapillary the hypha was surrounded by growth medium. A polyacrylamide gel was used as a substrate to facilitate the manipulation and contact of the microcapillaries with tips of growing hyphae without breaking the thin microcapillaries. We observed that it was possible to make the hyphae grow into the microcapillaries. Prior to 
(a)

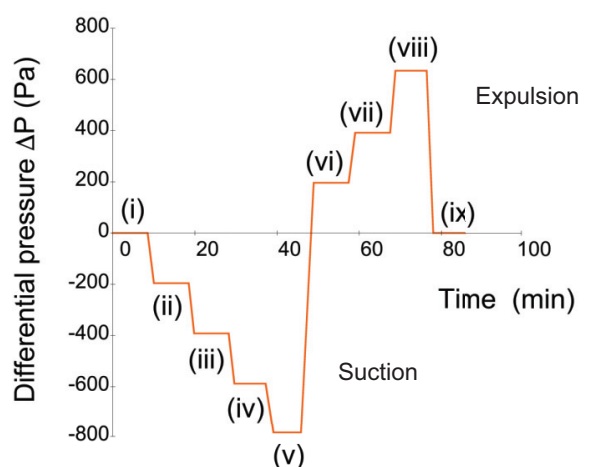

(b)
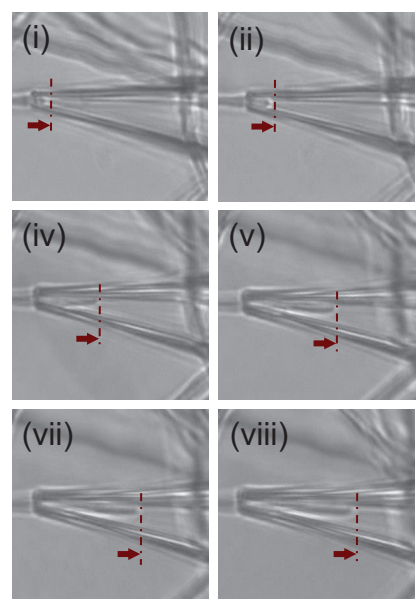
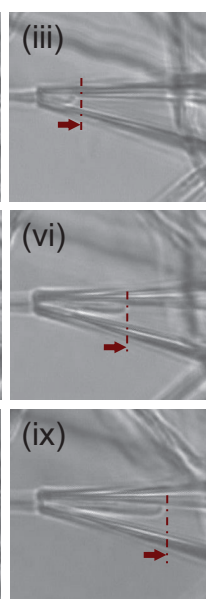

$10 \mu \mathrm{m}$

(c)

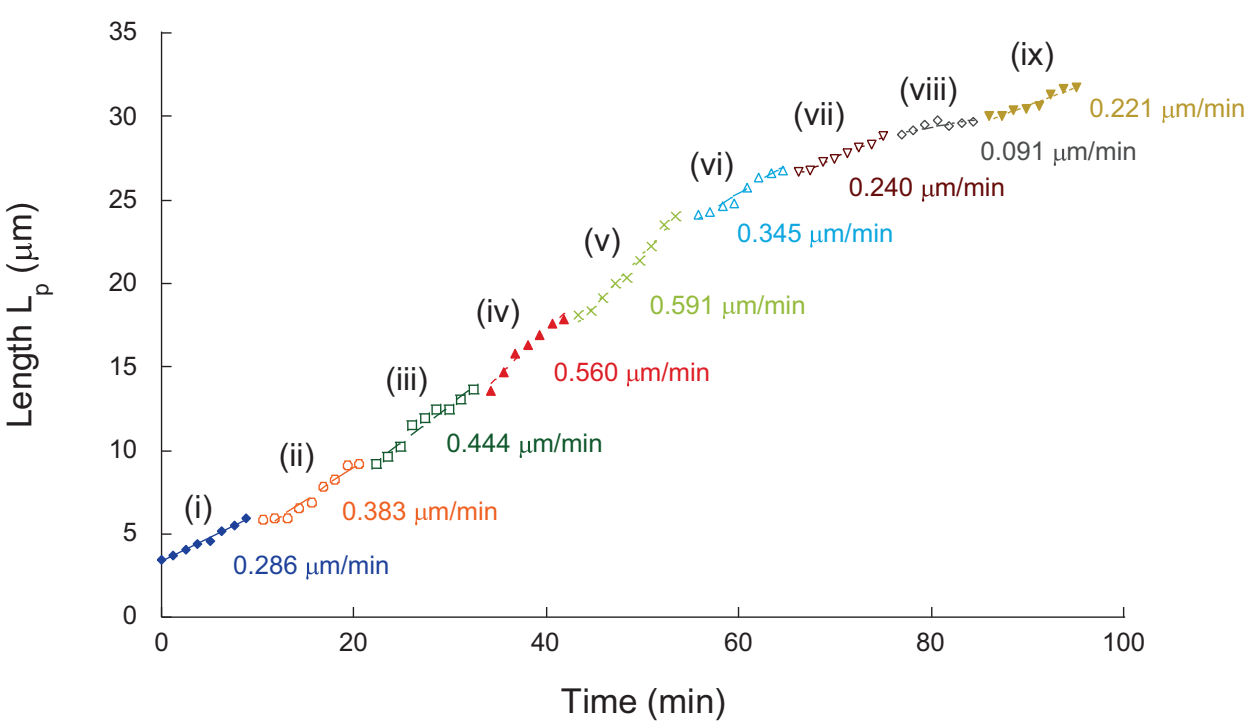

FIG. 2. Representative tip-pressure experiment of one hyphal tip. (a) Curve differential pressure $\Delta P$ versus time. The nine steps at different differential pressure are shown. (b) Microscopy images showing, for the nine steps of differential pressure, the length of the hypha inside the microcapillary, $L_{\mathrm{p}}$. (c) Curve length inside the microcapillary, $L_{\mathrm{p}}$, versus time; the slope of the curve during each loading step has been obtained by linear fitting.

measuring the progression of hyphae in these microcapillaries, we allowed them to enter and grow into the microcapillaries (with $\Delta P=0$ ) for a duration of $\sim 10 \mathrm{~min}$. Then we started the experiment, which included various steps of differential pressure $\Delta P$ (Fig. 2).

Figure 2 shows the curve aspirated length $L_{\mathrm{p}}$ versus time and the corresponding growth rates for a representative experiment. See Ref. [31]. It can be observed that the higher growth rates are obtained for the lower differential pressure $\Delta P$, as expected from the fact that the differential pressure indicates the value of the pressure opposing the progression of the cell tip. In the experiments, we used $\Delta P=0$ for the initial and for the final steps. As illustrated in Fig. 3, we obtained similar growth rates in both steps, indicating that the steady state is attained relatively rapidly for the characteristic times of the experiments, i.e., a duration of $\sim 10 \mathrm{~min}$ for each step and a total duration shorter than $100 \mathrm{~min}$ for the experiment.
Figure 3 shows the growth rate values obtained as the differential pressure was changed in discrete steps. A correlation between these two variables can be observed. A linear fitting

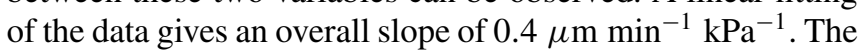
curve in Fig. 3 allows one also to estimate the differential pressure for which, during the characteristic times of $\sim 10 \mathrm{~min}$ used in the measurements, the cell stops growing: $\Delta P_{\text {stop }} \approx$ $650 \mathrm{~Pa}$.

\section{B. Mechanical model}

Since the duration of the experiments for a given value of differential pressure is relatively short, $10 \mathrm{~min}$, we considered it adequate to focus on a mechanical model for the analysis, assuming that metabolic and biochemical parameters are approximately constant during that time. In the analysis we assumed homogeneity and constancy of various parameters: the inner radius of the hypha, $r$; the osmotic pressure $\Delta \Pi$ 


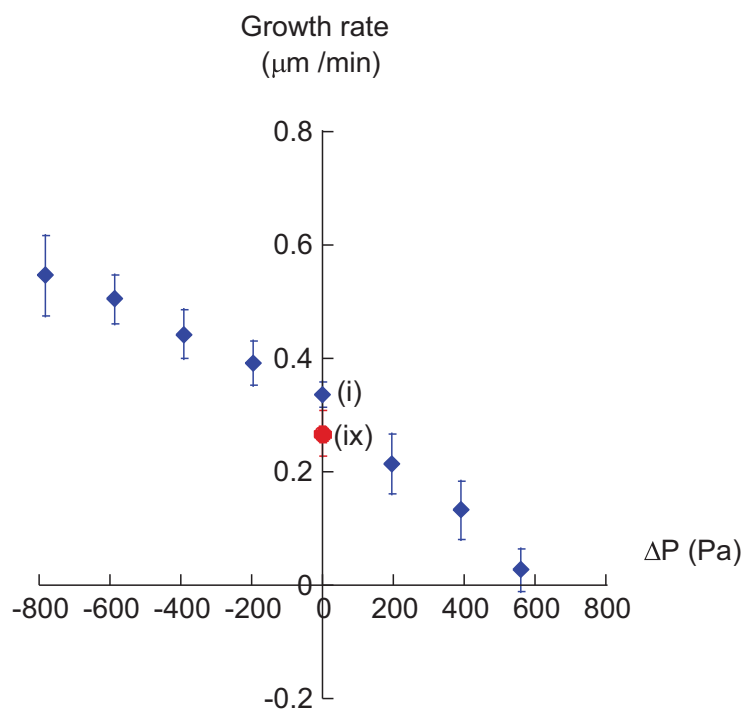

FIG. 3. Growth rate $d L_{\mathrm{p}} / d t$ versus differential pressure for the tip-pressure experiments. First (i) and last (ix) steps (both with $\Delta P=$ 0 ) are shown separately. Average values were obtained for $N=10$ experiments. Error bars represent standard error.

due to the different concentration of ions and molecules in the cell and in the growth medium; the hydraulic conductivity of the cell wall membrane for water, $L^{\text {wall }}$; and the viscosity of the aqueous medium inside the hypha, $\eta$. The model includes the flow of water through the cell wall, the Poiseuille flow of water inside the hypha, due to the gradient pressure, and the plastic deformation of the cell wall at the tip, which determines the hyphal length growth. A scheme of the model is shown in Fig. 4(a) and the details of the model are given in the Appendix.

The model predicts that the internal pressure in the hypha reaches its minimum value at the tip, decreasing exponentially from the asymptotic value (corresponding to the condition in which osmotic pressure balances the pressure difference between the interior of the cell and the surrounding medium), as represented in Fig. 4(b). The characteristic length for this exponential function, $\lambda$, depends on the internal viscosity of the cell, its diameter, and the water permeability of the wall.

As shown in the Appendix, the growth rate is given by

$$
\frac{d l}{d t}=\alpha-\beta \Delta P,
$$

where $d l / d t$ is the rate at which the hypha length $l$ varies along time $t ; \alpha$ and $\beta$ are parameters depending on the mechanical and flow properties in the hypha, with $\beta>0$. The hypha length $l$ is equal to the length inside the microcapillary, $L_{\mathrm{p}}$, plus the length outside the microcapillary; therefore, $d l / d t=d L_{\mathrm{p}} / d t$. A linear dependence between $d l / d t$ and $\Delta P$ is found: the lower $\Delta P$, the greater the growth rate $d l / d t$.

When the difference between pressure inside the cell and external pressure, $P_{\text {int }}^{\text {tip }}-P_{\text {ext }}^{\text {tip }}$, i.e., turgor pressure, is not greater than the threshold $Y$ to produce plastic stretching of the wall, the growth would stop. As shown in the Appendix, this condition is reached for a differential pressure $\Delta P_{\text {stop }}$ (a)

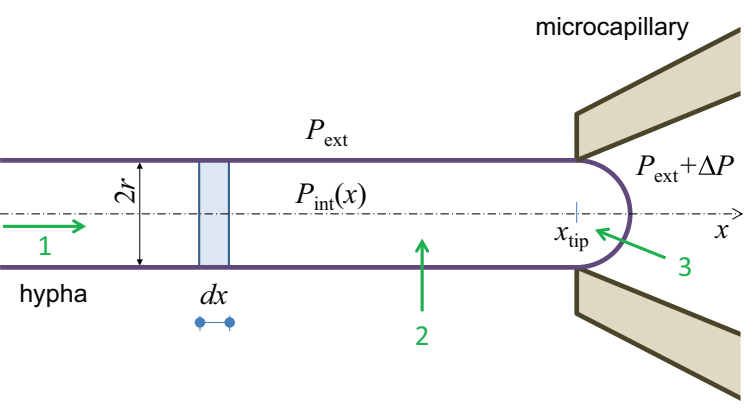

(b)

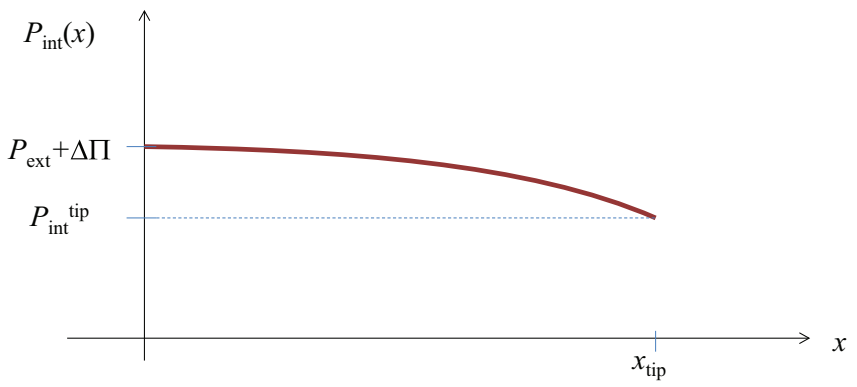

(c)

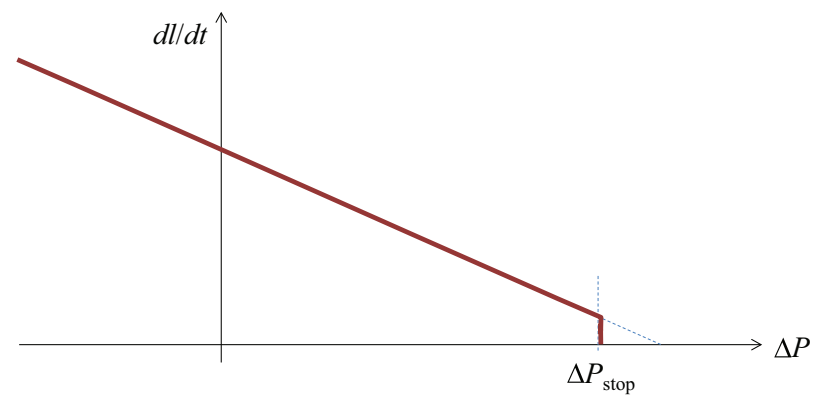

FIG. 4. Mechanical model. (a) Scheme of the hypha during the growth into the microcapillary, showing the main variables used in the analysis of the process. The three types of water flow are (1) internal water flow due to the pressure gradient (modeled using the Poiseuille equation), (2) flow through the hypha wall outside the microcapillary, and (3) inside the microcapillary, in both cases by osmosis. (b) The function for the internal pressure $P_{\text {int }}(x)$ varies exponentially; $\lambda$, represented schematically, is the characteristic length of the exponential decay (see Appendix). (c) Relationship between the growth rate $d l / d t$ and the differential pressure $\Delta P$ predicted by the model. It is found that $d l / d t \approx 0$ for $\Delta P_{\text {stop }}$ (see Discussion section).

given by

$$
\Delta P_{\text {stop }}=\frac{\alpha}{\beta}-\gamma
$$

Again, $\gamma$ is a function of the mechanical parameters, as defined in the Appendix. In Fig. 4(c) the proposed relationship between $d l / d t$ and $\Delta P$ is represented taking into account Eqs. (1) and (2).

The mechanical model does not take into account possible variations in the metabolism of the hyphae, which could produce changes in the osmotic pressure or in the deformability of the cell wall. Therefore, the model would be useful to explain changes in the growth ratio for moderately low 
duration, as we assume in our experiments. Besides, the model is a priori suited for a short length $L_{\mathrm{p}}$ of the hypha inside the microcapillary.

\section{Discussion}

The pressure difference between fungal cytoplasm and extracellular medium, i.e., turgor pressure, is balanced by the fungal cell wall, in contrast to the support provided by the contractile actin-myosin cytoskeleton in other eukaryotic cells $[32,33]$. Considering the fungal wall as a thin membrane of homogenous thickness, the highest value of the stress is found in the circumferential direction of the wall, because this stress is twice the stress in the longitudinal direction and in the semispherical tip of the hypha [34]. However, the cell growth takes place at the tip, where the material of the wall flows even if the predicted stress is smaller there, which is presumably due to the anisotropy and inhomogeneity of the wall [7]. This singular importance of the tip justifies the interest of the tip-pressure experiments.

The simple mechanical model, taking into account pressures and water flow and assuming homogeneity and constancy of various parameters, supports a nearly linear (if $\Delta P<$ $\left.\Delta P_{\text {stop }}\right)$ relationship between growth rate and differential pressure, which is in agreement with the experimental results (Fig. 3). The model predicts a differential pressure for which the growth stops, which is also observed in these experiments for $\Delta P_{\text {stop }} \approx 650 \mathrm{~Pa}$. The difference $P_{\text {int }}{ }^{\text {tip }}-P_{\text {ext }}{ }^{\text {tip }}$ must exceed the threshold $Y$ in order to produce plastic deformation of the wall and therefore that difference would be close to the threshold for the differential pressure of $650 \mathrm{~Pa}$.

Equation (2) may also be written as follows (see Appendix):

$$
\Delta P_{\text {stop }}=\left(1+4 \sqrt{\frac{\eta L^{\text {wall }}}{r}}\right)(\Delta \Pi-Y) .
$$

It is possible to estimate the order of magnitude of $\left(\eta L^{\text {wall }} / r\right)^{1 / 2}$ in Eq. (3), taking into account the following values for the parameters. The average viscosity of water in the cytoplasm is of the same order of magnitude of bulk water [35] and, furthermore, the viscosity of the cytoplasm is similar to that of water [36], $\eta \sim 1 \times 10^{-3} \mathrm{~Pa} \mathrm{~s}$ at $25^{\circ} \mathrm{C}$. The expected hydraulic conductivity of the wall-membrane layer is in the range $1 \times 10^{-14}-1 \times 10^{-12} \mathrm{~m} \mathrm{~Pa}^{-1} \mathrm{~s}^{-1}$ [37]. Therefore, for $r \sim 1 \times 10^{-6} \mathrm{~m},\left(\eta L^{\text {wall }} / r\right)^{1 / 2}<1 \times 10^{-4}$. This means that very approximately

$$
\Delta P_{\text {stop }} \approx \Delta \Pi-Y .
$$

Furthermore, using those numerical values, the estimated characteristic length $\lambda$ is of the order of meters [see Eq. (A8) in the Appendix] and then the variation of the internal pressure along the length of the hypha is relatively small. This is primarily due to the relatively low viscosity of water in the cytoplasm. This observation is in agreement with a previous estimation of the pressure gradient due to viscous flow in fungal hyphae, $0.01 \mathrm{kPa} / \mathrm{mm}$ [38]. The turgor pressure is between 0.4 and $0.8 \mathrm{MPa}[38,39]$. The internal pressure in the hypha is then nearly homogeneous and determined by the osmotic pressure:

$$
P_{\mathrm{int}} \approx P_{\mathrm{ext}}+\Delta \Pi
$$

Here we have neglected variations in the osmotic pressure. Using Eq. (5) and Eq. (A2) from the Appendix, the growth rate is found to be

$$
\frac{d l}{d t} \approx \phi(\Delta \Pi-Y-\Delta P) .
$$

Equation (6) is valid for $\Delta P<\Delta P_{\text {stop. }}$ This equation allows one to estimate $\phi$ as the slope for the experimental results in Fig. 3, i.e., $\phi \approx 7 \times 10^{-12} \mathrm{~m} \mathrm{~Pa}^{-1} \mathrm{~s}^{-1}$. This value, in turn, justifies Eq. (5), since it is deduced (using the previous numerical values) from the more complicated expression given in Eq. (A12) of the Appendix.

A differential pressure of $650 \mathrm{~Pa}$ is moderately small: it is of the order of $0.1 \%$ of the turgor pressure $(0.4-0.8 \mathrm{MPa}), 0.6 \%$ of the average atmospheric pressure at sea level, or $5 \%$ of a reference systolic manometric pressure of $100 \mathrm{~mm} \mathrm{Hg}$. The existence of a critical differential pressure $\Delta P_{\text {stop }}$ impeding the progression of the hypha tip suggests that controlling this parameter would be useful in the frame of fungal infections, for instance in the case of artery invasion. A local increase of $650 \mathrm{~Pa}$ for a distance of the order of $10 \mu \mathrm{m}$ would require a pressure gradient of $60 \mathrm{MPa} / \mathrm{m}$. Nevertheless, it would be difficult to find such a local gradient of pressure in the human body, where places with high gradients are located at the periphery of load bearing tissues like the bone or of pressurized cavities like the eye globe or the arteries. Although the progression of hyphal growth is obstructed by the effect of this local increase of pressure, some fungal species can invade blood vessels in aggressive infections [40]. Future experiments could test the fungal invasion arrest by the combined effect of pressure and medication.

Equation (4) indicates that the differential pressure required to stop the progression of the hypha tip is lower for lower osmotic pressure and for higher threshold $Y$. Therefore, treatments designed to approach these conditions could be tested using the proposed methodology, in order to confirm this expected trend and evaluate the effect on the hyphal growth. A treatment impeding the fungal invasion of arteries could be obtained following this rationale.

It is important to emphasize that the experiments were performed at a temperature of $25^{\circ} \mathrm{C}$, significantly lower than the optimal temperature for hyphal growth, $37^{\circ} \mathrm{C}$. A future study could provide information on the differential pressure required to arrest hyphal growth in physiological conditions and for long period.

\section{CONCLUSIONS}

The technique developed in this work, based on the micropipette aspiration technique, provides the possibility of studying the effect of the external pressure at the tip on the dynamics of hyphal growth. The procedure may be employed to assess the effect of different ambient conditions and the effect of the cell components of interest by studying mutant cells.

Studying the importance of the local conditions at the tip is relevant in the frame of fungal invasiveness. Therefore, this technique could provide clues for new treatments to fight fungal invasion of tissues where pressure is significantly different from the pressure in the surrounding tissues, as is the case in blood vessels. More studies, at physiological 
temperature and with infectious species, would be necessary to measure the differential pressure $\Delta P_{\text {stop }}$ required to impede invasion of tissues by these species, and to study possible treatments aiming to alter this value to obtain a therapeutic outcome.

\section{ACKNOWLEDGMENTS}

The authors received support from the Ministerio de Economía y Competitividad, Spain, througth Projects No. MAT2016-76847-R, No. BIO2015-30695R, and No. MAT2016-79832-R. B.G.-B. received a research grant from the Consejería de Educación, Juventud y Deporte de la Comunidad de Madrid.

B.G.-B. and Q.L. contributed equally to this work.

\section{APPENDIX}

During the growth process of one hypha, water and nutrients enter through the cell wall. In the following analysis we consider that the water flow and differences in pressure are the main contributions to describe our experiments in which we control the external pressure applied punctually at the tip of the hypha. This analysis is based on the study by Cosgrove [41] considering additionally the volume flow of the cytoplasm along the hypha [7].

The difference between internal pressure $P_{\text {int }}$ in the interior of the hypha and pressure in the surrounding medium, $P_{\text {ext }}$, is a determinant for the deformation of the cell wall required for the cell growth. Commonly it is assumed that the growth is due to the localized deformation at the tip of the hypha, where required materials are actively transported by means of molecular motors, and that the permanent-plastic-deformation requires a differential pressure exceeding a threshold value $Y$. The growth rate for the length of the hypha, $l$, may be written as

$$
\frac{d l}{d t}=\phi\left(P_{\mathrm{int}}{ }^{\mathrm{tip}}-P_{\mathrm{ext}}{ }^{\mathrm{tip}}-Y\right)+\frac{d}{d t} \Delta l_{\mathrm{el}},
$$

where $P_{\text {int }}{ }^{\text {tip }}-P_{\text {ext }}{ }^{\text {tip }}$ is the differential pressure at the tip and $\Delta l_{\mathrm{el}}$ represents the elastic extension of the hypha; $\phi$ is a growth rate coefficient related to the plastic deformation of the cell wall. Equation (A1) is valid for $P_{\text {int }}{ }^{\text {tip }}-P_{\text {ext }}$ tip $>Y$; otherwise, the differential pressure would be lower than the required threshold $Y$ and there would not be plastic deformation. For steady growth the main contribution to the rate is the plastic deformation and, therefore,

$$
\frac{d l}{d t} \approx \phi\left(P_{\text {int }}^{\text {tip }}-P_{\text {ext }}^{\text {tip }}-Y\right) .
$$

The threshold value $Y$ may be related to the stress required for the plastic deformation of the wall, $\sigma_{Y}$. For the semispherical shape of the hyphal-tip wall, the stress is given by $\sigma=\left(P_{\text {int }}{ }^{\text {tip }}-P_{\text {ext }}{ }^{\text {tip }}\right) 0.5 r / w$, where $r$ is the radius of the spherical wall and $w$ its thickness. Therefore, $Y=s_{\mathrm{Y}} 2 w / r$. In terms of force per unit length, or energy per unit surface (surface tension), required to increase the area of the wall, $T_{\mathrm{Y}}=s_{\mathrm{Y}} w$, the threshold value would be $Y=2 T_{\mathrm{Y}} / r$.

The larger ion concentration in the hypha results in a water flow through the cell wall. At a given point of the wall, the water volumetric flux $J_{\text {water }}^{\text {wall }}$, measured in volume per unit area and time, may be written as

$$
J_{\text {water }}^{\text {wall }}=L^{\text {wall }}\left(\Delta \Pi-\left(P_{\text {int }}-P_{\text {ext }}\right)\right),
$$

where $\Delta \Pi$ is the differential osmotic pressure between both sides of the wall and $L^{\text {wall }}$ the combined hydraulic conductivity of wall and membrane for water.

Inside the cell, water flows along the hypha to the tip due to the pressure gradient. The commonly used Poiseuille model assumes that inside the hypha the pressure $P_{\text {int }}(x)$ only depends on the axial position $x$ [Fig. 4(a)]. The model provides the following equation for the flow rate $Q_{\text {water }}^{\text {in }}$, i.e., the total volume of water per unit time through the cross section of the hypha:

$$
Q_{\text {water }}^{\text {in }}=\frac{\pi r^{4}}{8 \eta}\left(-\frac{d P_{\text {int }}(x)}{d x}\right) .
$$

The flow is oriented in the positive sense of the $x$ axis since the pressure decreases for growing values of $x$, i.e., $d P_{\text {int }}(x) / d x>0 ; r$ is the internal radius of the circular cross section of the hypha and $\eta$ is the dynamic viscosity of the aqueous fluid.

It is important to note that we are assuming a steady-state regime (see below) and, therefore, the pressure does not depend on the time.

The balance of mass between two sections separated by a distance $d x$ yields

$$
\begin{aligned}
& \frac{\pi r^{4}}{8 \eta}\left(-\frac{d P_{\text {int }}(x)}{d x}\right)+2 \pi r d x L^{\text {wall }}\left(\Delta \Pi-\left(P_{\text {int }}-P_{\text {ext }}\right)\right) \\
& \quad=\frac{\pi r^{4}}{8 \eta}\left[-\left(\frac{d P_{\text {int }}(x)}{d x}+\frac{d^{2} P_{\text {int }}(x)}{d x^{2}} d x\right)\right],
\end{aligned}
$$

where the fluid is assumed incompressible. From Eq. (A5) the following differential equation is obtained:

$$
\frac{d^{2} P_{\text {int }}(x)}{d x^{2}}=-\frac{16 \eta}{r^{3}} L^{\text {wall }}\left(\Delta \Pi-\left(P_{\text {int }}-P_{\text {ext }}\right)\right) .
$$

A solution for a stationary regime, assuming a sufficiently long hypha (i.e., the internal pressure far from the tip approaches asymptotically the value corresponding to osmotic equilibrium between both sides of the cell wall, $P_{\text {int }}=P_{\text {ext }}+$ $\Delta \Pi)$ may be straightforwardly obtained if $P_{\mathrm{ext}}, \Delta \Pi, \mathrm{r}, \eta$, and $L^{\text {wall }}$ are constant along the hypha:

$$
\begin{gathered}
P_{\text {int }}(x)=P_{\text {ext }}+\Delta \Pi+\left(P_{\text {int }}^{\text {tip }}-P_{\text {ext }}-\Delta \Pi\right) \exp \left(\frac{x-x_{\text {tip }}}{\lambda}\right) \\
\lambda=\sqrt{\frac{r^{3}}{16 \eta L^{\text {wall }}}},
\end{gathered}
$$

where $P_{\text {int }}{ }^{\text {tip }}$ is the internal pressure at the tip of the hypha. This result is valid along the hypha, with $-\infty \leqslant x \leqslant x_{\text {tip. }}$. $P_{\text {ext }}$ determines the water flow through the wall along the hypha length. Specifically, at $x=x_{\text {tip }}$, the external pressure $P_{\text {ext }}^{\text {tip }}=P_{\text {ext }}+$ $\Delta P$ applied through the microcapillary is different from $P_{\text {ext }}$.

To determine the value of the internal pressure at the tip, $P_{\text {int }}{ }^{\text {tip }}$, it is necessary to take into account the localized growing process at the tip. It requires the arrival of water equivalent to the newly created internal volume. The water arrives both from 
the interior of the hypha, pushed by the pressure gradient, and by permeation through the tip wall:

$$
\begin{aligned}
\pi r^{2} \frac{d l}{d t}= & \frac{\pi r^{4}}{8 \eta}\left(-\frac{d P_{\text {int }}(x)}{d x}\right)_{x=x_{\text {tip }}} \\
& +2 \pi r^{2} L^{\text {wall }}\left(\Delta \Pi-\left(P_{\text {int }}{ }^{\text {tip }}-P_{\text {ext }}{ }^{\text {tip }}\right)\right) .
\end{aligned}
$$

Second, the localized length growing needs the plastic stretching deformation of the wall [for which the materials brought by the cell are important, as expressed in Eq. (A2)].

Equating $d l / d t$ in Eqs. (A2) and (A9) and computing $\left(-\frac{d P_{\text {int }}(x)}{d x}\right)_{x=x_{\text {tip }}}$ from Eq. (A7), the following expression is obtained for the internal pressure at the tip:

$$
P_{\text {int }}^{\text {tip }}=\frac{a+b \Delta P}{c},
$$

where the constants $a, b$, and $c$ are given by

$$
\begin{aligned}
& a=\left(\frac{1}{2} \sqrt{\frac{L^{\text {wall }} r}{\eta}}+2 L^{\text {wall }}\right)\left(\Delta \Pi+P_{\mathrm{ext}}\right)+\phi\left(Y+P_{\mathrm{ext}}\right) \\
& b=2 L^{\text {wall }}+\phi \\
& c=\frac{1}{2} \sqrt{\frac{L^{\text {wall } r}}{\eta}}+2 L^{\text {wall }}+\phi
\end{aligned}
$$

Combining Eqs. (A7) and (A10), the expression for $P_{\text {int }}(x)$ may be written as

$$
\begin{aligned}
P_{\text {int }}(x)= & P_{\text {ext }}+\Delta \Pi+\left(\frac{a+b \Delta P}{c}-P_{\text {ext }}-\Delta \Pi\right) \\
& \times \exp \left(\frac{x-x_{\text {tip }}}{\lambda}\right) .
\end{aligned}
$$

Finally, combining Eqs. (A2) and (A10), the growth rate may be written as

$$
\frac{d l}{d t}=\phi\left(\frac{a}{c}-Y\right)-\phi\left(1-\frac{b}{c}\right) \Delta P .
$$

From this expression, Eq. (1) is obtained directly by renaming the constant terms. Therefore, a linear dependence between $d l / d t$ and $\Delta P$ is found. Since $(1-b / c)>0$, the growth rate $d l / d t$ is higher for lower external pressure at the tip, i.e., for lower $\Delta P$.

Equation (A13) is valid while the condition

$$
P_{\text {int }}^{\text {tip }}-P_{\text {ext }}^{\text {tip }}-\mathrm{Y} \geqslant 0
$$

is satisfied: the plastic stretching of the hypha wall is arrested if the difference between internal and external pressure is not higher than the threshold $Y$. The differential pressure $\Delta P_{\text {stop }}$ for which $P_{\text {int }}{ }^{\text {tip }}-P_{\text {ext }}{ }^{\text {tip }}-\mathrm{Y}=0$ is then

$$
\Delta P_{\text {stop }}=\frac{a-c\left(Y+P_{\text {ext }}\right)}{c-b}=\left(1+\frac{16 \eta \lambda L^{\text {wall }}}{r^{2}}\right)(\Delta \Pi-Y) \text {. }
$$

From Eq. (A15), Eq. (2) is obtained by using $\gamma=$ $c P_{\text {ext }} /(c-b)$ and Eq. (3) by using Eq. (A8). Equation (A13) allows one to determine also the differential pressure at the tip that would be required to reach $d l / d t=0$ without satisfying condition (A14):

$$
\Delta P_{0}=\frac{a / c-Y}{1-b / c}=\Delta P_{\mathrm{stop}}+\frac{8 \eta \lambda}{r^{2}} c P_{\mathrm{ext}} .
$$

Equation (A16) shows that $\Delta P_{\text {stop }}<\Delta P_{0}$. In the discussion section we justify that for the experimental values $\Delta P_{\text {stop }} \approx$ $\Delta P_{0}$.
[1] G. Gooday, Mycol. Res. 99, 385 (1995).

[2] L. Zhao, D. Schaefer, H. Xu, S. J. Modi, W. R. LaCourse, and M. R. Marten, Biotechnol. Prog. 21, 292 (2005).

[3] S. Yano, K. Kobayashi, S. Shishido, and H. Nakano, Intern. Med. 38, 372 (1999).

[4] J. E. Galagan, S. E. Calvo, C. Cuomo, L. Ma, J. R. Wortman, S. Batzoglou, S. Lee, M. Baştürkmen, C. C. Spevak, and J. Clutterbuck, Nature (London) 438, 1105 (2005).

[5] N. Taheri-Talesh, T. Horio, L. Araujo-Bazan, X. Dou, E. A. Espeso, M. A. Penalva, S. A. Osmani, and B. R. Oakley, Mol. Biol. Cell 19, 1439 (2008).

[6] J. Latge, Mol. Microbiol. 66, 279 (2007).

[7] R. R. Lew, Nat. Rev. Microbiol. 9, 509 (2011).

[8] T. Horio and B. R. Oakley, Mol. Biol. Cell 16, 918 (2005).

[9] Y. Chebli, J. Kroeger, and A. Geitmann, Mol. Plant. 6, 1037 (2013).

[10] J. H. Kroeger, R. Zerzour, and A. Geitmann, PloS One 6, e18549 (2011).

[11] S. Jackson and I. Heath, Microbiol. Rev. 57, 367 (1993).

[12] G. S. Worthen, B. Schwab 3rd, E. L. Elson, and G. P. Downey, Science 245, 183 (1989).

[13] J. D. Pajerowski, K. N. Dahl, F. L. Zhong, P. J. Sammak, and D. E. Discher, Proc. Natl. Acad. Sci. USA 104, 15619 (2007).
[14] J. Guck, S. Schinkinger, B. Lincoln, F. Wottawah, S. Ebert, M. Romeyke, D. Lenz, H. M. Erickson, R. Ananthakrishnan, and D. Mitchell, Biophys. J. 88, 3689 (2005).

[15] H. Vogler, D. Felekis, B. J. Nelson, and U. Grossniklaus, Plants 4, 167 (2015).

[16] J. H. Kroeger, A. Geitmann, and M. Grant, J. Theor. Biol. 253, 363 (2008).

[17] F. Chang and K. C. Huang, BMC Biol. 12, 54 (2014).

[18] J. K. E. Ortega and S. W. J. Welch, Math. Modell. Nat. Phenom. 8, 35 (2013).

[19] A. Boudaoud, Phys. Rev. Lett. 91, 018104 (2003).

[20] A. Goriely and M. Tabor, J. Theor. Biol. 222, 211 (2003).

[21] J. H. Kroeger, F. B. Daher, M. Grant, and A. Gieitmann, Biophys. J. 97, 1822 (2009).

[22] M. Riquelme and E. Sánchez-León, Curr. Opin. Microbiol. 20, 27 (2014).

[23] R. J. Dyson and O. E. Jensen, J. Fluid Mech. 655, 472 (2010).

[24] A. Geitmann, Am. J. Bot. 93, 1380 (2006).

[25] R. M. Hochmuth, J. Biomech. 33, 15 (2000).

[26] G. R. Plaza, N. Marí, B. G. Gálvez, A. Bernal, G. V. Guinea, R. Daza, J. Pérez-Rigueiro, C. Solanas, and M. Elices, Phys. Rev. E 90, 052715 (2014). 
[27] B. Sabass, M. L. Gardel, C. M. Waterman, and U. S. Schwarz, Biophys. J. 94, 207 (2008).

[28] D. J. Cove, Biochim. Biophys. Acta 113, 51 (1966).

[29] M. Penalva, Fungal Genet. Biol. 42, 963 (2005).

[30] G. Esteban-Manzanares, B. Gonzalez-Bermudez, J. Cruces, M. De la Fuente, Q. Li, G. V. Guinea, J. Perez-Rigueiro, M. Elices, and G. R. Plaza, Ann. Biomed. Eng. 45, 1375 (2017).

[31] See Supplemental Material at http://link.aps.org/supplemental/ 10.1103/PhysRevE.96.022402 for a list of the experimental rates and for a video showing the hyphal growth in one tip-pressure experiment.

[32] G. R. Plaza and T. Q. P. Uyeda, Soft Matter 9, 4390 (2013).

[33] G. R. Plaza, T. Q. P. Uyeda, Z. Mirzaei, and C. A. Simmons, Soft Matter 11, 5435 (2015).
[34] A. Geitmann and J. K. E. Ortega, Trends Plant Sci. 14, 467 (2009).

[35] K. Luby-Phelps, Int. Rev. Cytol. 192, 189 (1999).

[36] K. Fushimi and A. Verkman, J. Cell Biol. 112, 719 (1991).

[37] C. Maurel, Annu. Rev. Plant Physiol. Plant Mol. Biol. 48, 399 (1997).

[38] R. Lew, Microbiology 151, 2685 (2005).

[39] R. Howard and B. Valent, Annu. Rev. Microbiol. 50, 491 (1996).

[40] W. Li, N. Shafi, R. Periakaruppan, T. Valyi-Nagy, J. Groth, and F. D. Testai, J. Stroke Cerebrovasc. Dis. 24, E39 (2015).

[41] D. Cosgrove, Annu. Rev. Plant Physiol. Plant Mol. Biol. 37, 377 (1986). 